\title{
Dermagraft: Use in the Treatment of Chronic Wounds
}

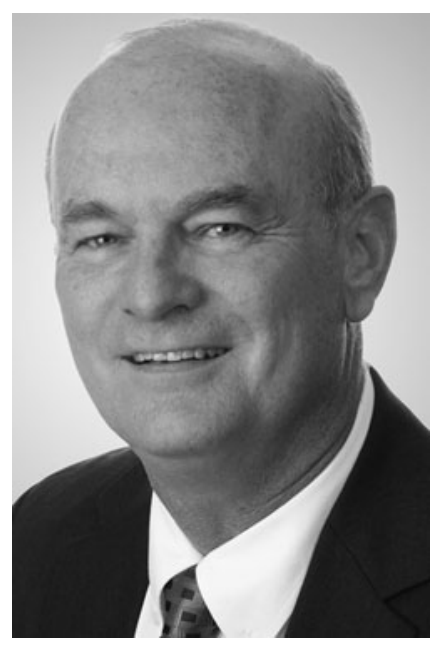

Charles E. Hart

Submitted for publication April 6, 2011

${ }^{*}$ Correspondence: Advanced BioHealing, Inc. 214 Overlook Circle, Suite 205, Brentwood, TN 37027 (e-mail: chart@abh.com).

\section{Abbreviations and Acronyms \\ $\mathrm{DFU}=$ diabetic foot ulcer \\ $\mathrm{FDA}=$ Food and Drug \\ Administration \\ VLU $=$ venous leg ulcer}

\author{
Charles E. Hart, ${ }^{*}$ Andrea Loewen-Rodriguez, and Jan Lessem \\ Advanced BioHealing, Inc., Brentwood, Tennessee.
}

Problem: Lower limb ulceration is a common problem in clinical practice. A variety of metabolic and physical causes can lead to a diversity of chronic ulcer types, including diabetic foot ulcers (DFUs) and venous leg ulcers (VLUs).

Solution: A wide variety of technologies have been developed to treat chronic wounds, with varying levels of success. Depending upon the type and severity of the wound being treated, treatments may include systemic or local antibiotic therapy, application of fillers such as collagen sponges, use of negative wound pressure, hyperbaric oxygen therapy, application of select growth factors, advanced wound dressings, and more recently, the use of cell-based tissueengineered products.

New Technology: Dermagraft ${ }^{\circledR}$ is a sterile, cryopreserved, human fibroblastderived dermal substitute generated by the culture of neonatal dermal fibroblasts onto a bioabsorbable polyglactin mesh scaffold. During the product-manufacturing process, the human fibroblasts proliferate to fill the interstices of this scaffold and secrete collagen, other extracellular matrix proteins, growth factors, and cytokines, creating a three-dimensional human tissue containing metabolically active living cells.

Indications for Use: Dermagraft has been approved for marketing in the United States for the treatment of DFUs. In addition, the product is in active development for the treatment of VLUs and has been clinically used in a variety of other indications to stimulate wound healing.

Caution: When treating DFUs, Dermagraft should be used in conjunction with standard wound care regimens and in patients who have adequate blood supply to the involved foot.

\section{UNMET NEED}

TWO PRIMARY TYPES of chronic ulcers include diabetic foot ulcers (DFUs) and venous leg ulcers (VLUs). A key factor in the development of a DFU is neuropathy, leading to the inability to feel pain, which normally serves as a protective sensation. This combined with repetitive excessive pressure over bony prominences can lead to tissue breakdown and ulceration. If the ulcer goes untreated, it can lead to severe infection and, ultimately, amputation of the lower limb. The principal cause of
VLU is ambulatory hypertension resulting from valvular incompetence and venous reflux. Ulcers of this type can last for years with associated morbidities including pain, infection, and ulcer odor, leading to social isolation.

\section{PRODUCT TECHNOLOGY}

Dermagraft is a sterile, cryopreserved, human fibroblast-derived dermal substitute generated by the culture of neonatal dermal fibroblasts onto a bioresorbable polyglactin mesh 
scaffold (Fig. 1). During the manufacturing process, the human fibroblasts proliferate to fill the interstices of this scaffold and secrete collagen, other extracellular matrix proteins, growth factors, and cytokines, creating a three-dimensional humanderived dermal substitute containing metabolically active, living cells. ${ }^{1,2}$ Dermagraft contains only human dermal fibroblasts and their secreted products and is devoid of other cell types also found in skin (e.g., macrophages, lymphocytes, endothelial cells, or keratinocytes). The fibroblasts are from a qualified cell bank and have been extensively screened for infectious agents. The polyglactin mesh that supports the fibroblasts has been tested and approved for use in humans and is a commonly used suture material.

\section{INNOVATION}

In treating a DFU or VLU, debridement of necrotic/nonviable tissue results in a healthier wound bed. After ulcer debridement, cell-based tissue-engineered agents such as Dermagraft may be applied to the wound.
In DFUs and VLUs, the normal healing process is arrested. The use of regenerative medicine approaches to enhance the wound healing responses has gained increased acceptance over the past decade, with clinical benefits demonstrated through randomized controlled trials. Dermagraft delivers a collagen-rich living human dermal matrix to the prepared ulcer wound bed. ${ }^{2}$ Metabolically active fibroblasts are distributed throughout the Dermagraft and retain the capacity for secreting a variety of regulatory and structural proteins. Dermagraft is designed to take part in and promote the formation of normal wound tissue, and clinical trials have demonstrated the efficacy of the product to improve the rate of healing of DFUs when compared with standard bandaging regimens. ${ }^{3}$

\section{PEER-REVIEWED DATA}

Dermagraft was investigated for the treatment of DFUs in a Phase 3, randomized, controlled clinical study that included 35 centers within the United States. ${ }^{3}$ Inclusion criteria included the patient having type I or II diabetes, with a plantar

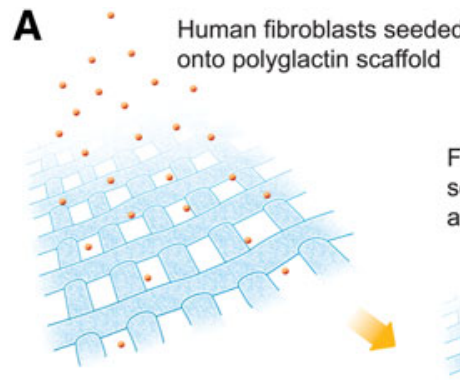
End product contains well
developed extracellular matrix
with living fibroblasts

Fibroblasts multiply and secrete growth factors and matric proteins
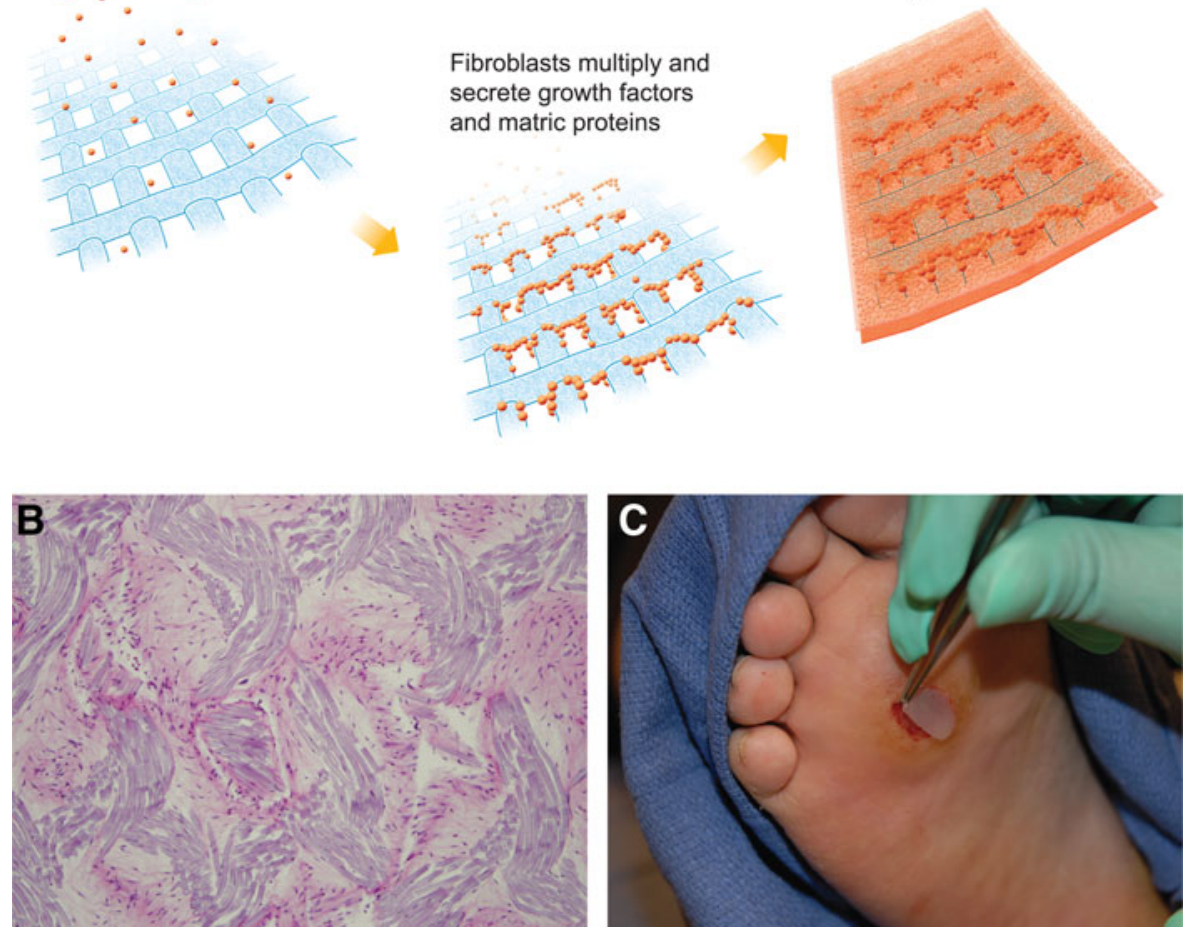

Figure 1. (A) Dermagraft is generated by seeding human dermal fibroblasts onto a polyglactin mesh scaffold. The cells are allowed to expand during which time they lay down a three-dimensional matrix containing collagens type I, III, and VII, various growth factors and cytokines, and numerous proteoglycans. The final product, which contains living human cells, is cryopreserved for storage. (B) Transverse section of Dermagraft stained with hematoxylin and eosin. The stain emphasizes the general morphology of the tissue, depicting human fibroblasts distributed throughout the naturally secreted collagen matrix and interposed between the polyglactin strands (magnification, $\times 200$ ). (C) Placement of Dermagraft onto a diabetic foot ulcer. 
DFU size between 1 and $20 \mathrm{~cm}^{2}$ without muscle, bone, tendon, or joint capsule exposure. If the study ulcer in the patient had not decreased in size by $50 \%$ after 2 weeks of standard therapy, the patient was randomized to either the Dermagraft treatment group $(n=130)$ or control $(n=115)$ (conventional wound care therapy). The study ulcers were similarly treated in both study groups except for the administration of Dermagraft. Patients in the Dermagraft group received weekly applications for up to 8 weeks as necessary, over the course of the 12week study. All patients in both the treatment and control groups received pressure-reducing foot wear.

Evaluations of both the control and Dermagraft treatment groups were weekly made until either complete wound closure was observed or the patients reached the week 12 visit without evidence of wound closure. The study results demonstrated that patients with chronic DFUs of $>6$ weeks duration before entrance into the study experienced a significant clinical benefit when treated with Dermagraft versus patients treated with conventional therapy alone. The primary endpoint of the study was complete wound closure, defined as "full epithelialization of the wound with the absence of drainage." The study results showed that there was a significant increase $(p=0.023)$ in the percentage of wounds healed by 12 weeks with Dermagraft treatment (30\%) when compared with the control group (18.3\%), ${ }^{3}$ corresponding to a $64 \%$ increase in the proportion of DFUs that headed with Dermagraft treatment (Fig. 2). The results from the study further demonstrated that the Dermagraft group had a significantly faster time to complete wound closure than the control group ( $p=0.04)$.

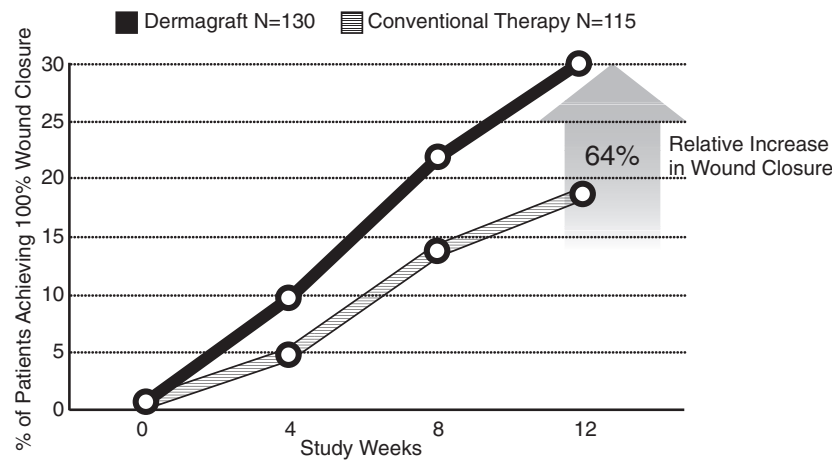

Figure 2. Comparison of patients treated with Dermagraft versus those receiving conventional therapy for healing of diabetic foot ulcers. The study results demonstrated that at 12 weeks after initiation of therapy, Dermagraft treatment led to a $64 \%$ increase in the proportion of patients exhibiting complete wound closure when compared with patients receiving conventional therapy.
Although overall adverse events were not significantly different between the two study groups, the percentage of patients who developed study ulcer-related adverse events was significantly lower in the Dermagraft-treated group $(p=0.007) .^{3}$

The clinical use of Dermagraft to stimulate wound healing has been reported in the literature for a variety of indications beyond DFUs, including the treatment of dystrophic epidermolysis bullosa, ${ }^{4}$ chronic surgical wounds, ${ }^{5}$ and wound dehiscence after amputations. ${ }^{6}$ Additionally, nonclinical data demonstrating the pro-angiogenic activity of Dermagraft support its use for wound healing applications and suggest its potential use in cardiovascular indications. ${ }^{7}$

These reports highlight the potential value of cell-based tissue-engineered products such as Dermagraft to positively impact healing in a wide variety of nonhealing indications. Apligraf, a second cell-based therapeutic, has received premarket approval by the U.S. Food and Drug Administration (FDA) for the treatment of DFUs and VLUs (reviewed by Ehrenreich and Ruszczak ${ }^{8}$ ).

\section{NON-PEER-REVIEWED OBSERVATIONS Current clinical studies}

Dermagraft is being developed for the treatment of VLU and is currently in a worldwide clinical trial, initiated in 2009, comparing Dermagraft used in conjunction with four-layer compression bandaging therapy versus four-layer compression bandaging therapy alone. A pilot study conducted with Dermagraft for this indication suggested that its use leads to improved healing. ${ }^{9}$

\section{SUMMARY ILLUSTRATION}

The tissue engineered agent Dermagraft addresses multiple aspects of tissue regeneration (the need for cells, signaling molecules, and scaffolds) in order to provide an optimal therapeutic approach for regenerative medicine therapies.

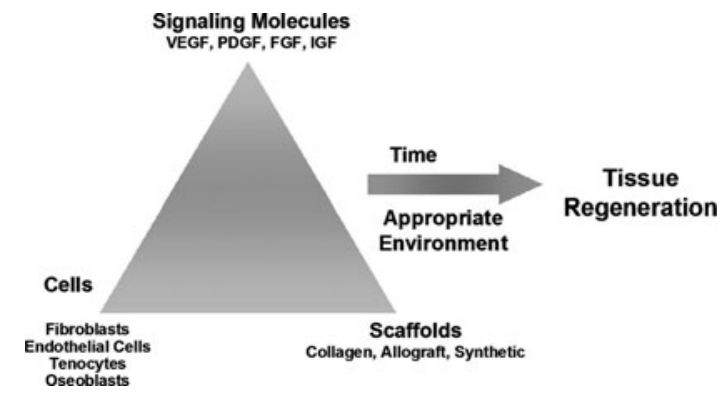




\section{CAUTION, CRITICAL REMARKS, AND RECOMMENDATIONS}

Dermagraft has been approved by the U.S. FDA as a Class III medical device for use in the treatment of full-thickness DFUs of $>6$ weeks duration, which extend through the dermis, but without tendon, muscle, joint capsule, or bone exposure. A consensus panel ${ }^{10}$ recently provided a series of recommendations for the treatment of DFUs, highlighting the use of advanced cellular therapies such as Dermagraft in conjunction with standard wound care regimens.

\section{ACKNOWLEDGMENTS AND FUNDING SOURCES}

The authors acknowledge Susan Weinbach, Carmen Perez, Jenna Curammeng, Kerry QuinnSenger, and Lisa DeBoer for chapter review and technical assistance.

\section{AUTHOR DISCLOSURE AND GHOSTWRITING}

C.H., A.L.R., and J.L. are employees of Advanced BioHealing. No ghostwriters were used to write this article.

\section{REFERENCES}

1. Mannsbridge JN, Liu K, Pinney RE, Patch $R$, Ratcliffe $A$, and Naughton GK: Growth factors secreted by fibroblasts: role in healing diabetic foot ulcers. Diabetes Obesity Metab 1999; 1: 265.

2. Naughton GK, Mansbridge JN, and Gentzkow G: A metabolically active human dermal replacement for the treatment of diabetic foot ulcers. Artif Organs 1997; 21: 1203.

3. Marston WA, Hanft J, Norwood P, and Pollak R The efficacy and safety of Dermagraft in improving the healing of chronic diabetic foot ulcers: results of a prospective randomized trial. Diabetes Care 2003; 26: 1701.

4. Sibbald RG, Zuker R, Coutts P, Coelho S, Williamson $\mathrm{D}$, and Queen $\mathrm{D}$ : Using a dermal skin substitute in the treatment of chronic wounds secondary to recessive dystrophic epidermolysis bullosa: a case series. Ostomy Wound Manage 2005; 51: 22.

5. Joncas V, Prado R, Price Ritchie C, Kaufman LM, Schober-Flores $\mathrm{C}$, and Arbuckle HA: The closure of a large chronic abdominal wound in a neonate utilizing a biologic dressing. Adv Skin Wound Care 2010; 23: 404

6. Talarico RH, Kim J, and Lin D: The use of human fibroblast derived dermis on post-surgical wound dehiscence: a report of two cases. J Diabetic Foot Complications 2010; 2: 28.

7. Fitzpatrick JR 3rd, Frederick JR, McCormick RC, Harris DA, Kim AY, Muenzer JR, Gambogi AJ, Liu JP, Paulson EC, and Woo YJ: Tissue-engineered pro-angiogenic fibroblast scaffold improves myocardial perfusion and function and limits ventricular remodeling after infarction. J Thoras Cardiovasc Surg 2010; 140: 667.

8. Ehrenreich M, and Ruszczak Z: Update on tissueengineered biological dressings. Tissue Eng 2006; 12: 2407.

9. Omar AA, Mavor, AID, Jones AM, and HomerVanniasinkam S: Treatment of venous leg ulcers with Dermagraft. Eur J Vasc Endovasc Surg 2004; 27: 666 .

10. Snyder RJ, Kirsner RS, Warriner III RA, Lavery LA, Hanft JR, and Sheehan P: Consensus recommendations on advancing the standard of care for treating neuropathic foot ulcers in patients with diabetes. Ostomy Wound Manage 2010; 56: S1. 